\title{
Circulating levels of irisin is elevated in hypothyroidism, a case-control study
}

Insan Ateş', Mustafa Altay', Canan Topçuoğlu², Fatma Meriç Yılmaz²

\begin{abstract}
Objective: Our objective in this study was to determine the relationship between irisin hormone, which has a similar effect with thyroid hormones on adipose tissue and the metabolism, and the thyroid functions and the obesity secondary to thyroid disease. Subjects and methods: Seventy-four patients were included in the study, of the patients, 37 were newly diagnosed with Hashimoto's thyroiditis related hypothyroidism but not started on a treatment yet, and the remaining 37 were healthy volunteers without a known disease. Serum thyroid stimulating hormone (TSH), free thyroxin (fT4), anti-thyroglobulin and anti-thyroid peroxidase were measured and thyroid ultrasonography was performed in both groups. Serum irisin levels were measured using the commercially available ELISA kit. Results: The hypothyroidism group had higher levels of irisin compared to the control group ( $2.77 \mathrm{ng} / \mathrm{mL}$ vs. $2.15 \mathrm{ng} / \mathrm{mL}$ respectively; $p=0.017)$. The hypothyroidism group had higher median levels of irisin in the obese patients than those in the control group $(3.10 \mathrm{ng} / \mathrm{mL} v s .2 .10 \mathrm{ng} / \mathrm{mL}$ respectively; $p=0.013$ ). Irisin level was negatively correlated with age in the whole population and patients with hypothyroidism ( $r=-0.255, p=0.028 ; r=-0.346, p=0.036$ respectively). Irisin level was positively correlated with TSH $(r=0.247, p=0.034)$ but negatively correlated with the fT4 $(r=-0.316, p$ $=0.006$ ) in the whole population. Obesity, $\mathrm{fT} 4$ and irisin levels were identified to be independent predictors in the diagnosis of hypothyroidism in the multivariable logistic regression analysis. Conclusion: To the best of our knowledge, this study is the first in literature to identify that obesity, irisin level and fT4 level are independent risk factors for hypothyroidism. Arch Endocrinol Metab. 2016;60(2):95-100
\end{abstract}

Keywords

FNDC5; Hashimoto's thyroiditis; hypothyroidism; PPAR $\gamma$; thyroid
${ }^{1}$ Ankara Numune Education and Research Hospital, Department of Internal Medicine, Ankara, Turkey ${ }^{2}$ Ankara Numune Education and Research Hospital, Department of Biochemistry, Ankara, Turkey

Correspondence to: İhsan Ateş

Ankara Numune Education and Research Hospital, Department of Internal Medicine, Sinhiye, Ankara, Turkey dr.ihsanates@hotmail.com

\section{INTRODUCTION}

$\mathrm{H}$ ashimoto's thyroiditis (HT) is characterized by the destruction of thyroid follicular cells at different levels that results in the formation of thyroid autoantibodies and development of hypothyroidism. HT is the most common cause of the overt and subclinical hypothyroidism in clinics (1). In the overt hypothyroidism phase, many metabolic processes in the body, such as differentiation in the adipose tissue, are affected as a result of inadequate synthesis of thyroid hormones. Increased thyroid stimulating hormone (TSH) causes adipogenesis by stimulating preadiposite differentiation directly through the receptors in the adipose tissue (2). Fatty tissue is increased by adipocyte hypertrophy, which is a result of previous adipogenesis, and newly formed adipocyte hypertrophy (3), which results in clinical obesity. This mechanism is not the only explanation for the relationship between thyroid hormones and obesity.
Hormones synthesized in other endocrine organs are also known to have effects on the adipose tissue (4).

Recently, the relationship of irisin hormone, discovered by Boström and cols. in 2012 (5), with obesity and other hormones has often been a subject of study $(6,7)$. Irisin is secreted as a product of fibronectin type III domain containing 5 (FNDC5) in the human and mice skeletal muscle. FNDC5 is secreted by peroxisome proliferator-activated receptor $\gamma$ (PPAR $\gamma$ ) coactivator $1 \alpha(\mathrm{PGCl}-\alpha)$ after an exercise (8). Irisin can be synthesized in many tissues other than skeletal muscle (9) and replaces the white fatty tissue with brown fatty tissue, resulting in thermogenesis and energy consumption. FNDC5, which is the precursor of irisin was shown to be present in many tissues, including the thyroid tissue (10). Although its relationship with many diseases, such as type 2 diabetes mellitus, metabolic syndrome, insulin resistance, chronic renal disease, and anorexia nervosa 
has been investigated, its association with the thyroid functions tests (TFT) in HT patients with hypothyroidism remains unknown.

Our objective in this study was to determine the relationship between irisin hormone, which has a similar effect with thyroid hormones on adipose tissue and the metabolism, and the thyroid functions and the obesity secondary to thyroid disease.

\section{SUBJECTS AND METHODS}

This study was conducted at Internal Medicine Clinic of Ankara Numune Training and Research Hospital between February 2014 and June 2014. The study was in compliance with the Declaration of Helsinki and was approved by the Local Ethics Research Committee. All subjects provided their written informed consent prior to participating in the study.

A total of seventy-four patients were included in the study, who were over the age of 18 years. Of the patients, 37 were newly diagnosed with HT dependent hypothyroidism but not started on a treatment yet, and the remaining 37 were healthy volunteers without a known disease. The exclusion criteria included hypertension, dyslipidemia (low density lipoprotein (LDL) cholesterol $>130 \mathrm{mg} / \mathrm{dL}$, triglycerides $(\mathrm{TG})>150 \mathrm{~g} / \mathrm{dL}$ ), diabetes mellitus, acute-chronic renal disease, nephritic-range proteinuria, coronary heart disease, heart failure, peripheral artery disease, cerebrovascular event, malignancy, liver diseases, rheumatic diseases, alcohol intake, and smoking.

Hashimoto's thyroiditis was diagnosed by high levels of anti-thyroglobulin (anti-TG) and/or anti-thyroid peroxidase (anti-TPO) in serum and/or the presence of heterogeneous echogenicity in the thyroid parenchyma shown by thyroid ultrasonography (USG). Hypothyroidism was diagnosed by high levels of TSH (normal range: $0.5-4 \mu \mathrm{IU} / \mathrm{mL}$ ) and low levels of free thyroxin (fT4) (normal range: $0.9-1.7 \mathrm{ng} / \mathrm{dL}$ ). Serum TSH, fT4, anti$\mathrm{TG}$, and anti-TPO levels of those in the control group were measured and HT was excluded by thyroid USG.

Two blood pressure measurements were done $5 \mathrm{mi}$ nutes apart after 5 minutes of rest for each participant during outpatient clinic visits. Blood pressure was determined by calculating the average of 2 mesaurements. Omron M3 (Omron Healthcare Co. Ltd. Japan) digital automatic blood pressure monitor calibrated according to European Society of Hypertension International Protocol 2002 ve 2010 was used for the measurements.
Following an 8-hour fasting, blood samples were obtained in the morning for biochemical parameters. Samples were quickly centrifuged at $4000 \mathrm{rpm}$ for 10 minutes to separate the plasma and serum, and the serum samples were kept at $-80^{\circ} \mathrm{C}$. Following this, all parameters were studied in the same serum sample.

Thyroid function tests were analyzed at the hormone laboratory using Electro-chemiluminescence immunoassay. Total cholesterol and TG levels were measured through enzymatic colorimetric method; and the high density lipoprotein cholesterol (HDL-C) was measured by homogenous enzymatic colorimetric method using Hitachi Modular P800 (Roche Diagnostics Corp. Indiana, USA) auto-analyzer. Low density lipoprotein cholesterol (LDL-C) was calculated using the Friedewald method (11).

Serum irisin levels were measured using the commercially available ELISA kit (EASTBIOPHARM, Hangzhou Eastbiopharm Co. Ltd. China, REF: CKE90905, LOT: 201402). The measurement range was 0.5-30 ng/mL.

\section{Statistical analyses}

Statistical Package for Social Sciences (SPSS) for Windows 20 (IBM SPSS Inc., Chicago, IL) program was used for the statistical analysis. Kolmogorov-Smirnov test was used to determine data with normal distribution. Numeric variables with normal distribution were expressed in mean \pm standard deviation, and numeric variables without normal distribution were expressed in median. Categorical variables were presented in figures and percentage. Spearman correlation analysis was performed for the correlation between the numeric parameters. T test (for numeric variables with normal distribution) and Mann Whitney U Test (for numeric variables without normal distribution) were used to identify the risk factors, which differed in two groups, in independent samples. Chi- Squara Test and Fisher's Final Chi-Square Test were used to compare the categorical data. Multivariable logistic regression analysis was used to identify the independent predictors that have an effect on the presence of hypothyroidism. p $<0.05$ value was considered significant in statistical analyses.

\section{RESULTS}

A total of 74 patients were included in the study, $37 \mathrm{pa}-$ tients with hypothyroidism ( 4 males, 33 females, mean age: $32.27 \pm 8.7$ years) and 37 volunteers (1 male, 36 females, mean age: $34.76 \pm 11.9$ years) for the healthy 
control group. Both groups had similar demographical characteristics such as age, gender, and BMI (Table 1). Systolic blood pressure $(127.38 \pm 9.7 \mathrm{mmHg} v s .120 .22$ $\pm 11.2 \mathrm{mmHg}$, respectively, $\mathrm{p}=0.004)$, diastolic blood pressure $(65.97 \pm 8.5 \mathrm{mmHg}$ vs. $60.0 \pm 9.7 \mathrm{mmHg}$, respectively; $\mathrm{p}=0.006$ ), and the proportion of obese patients $(40.5 \%$ vs. $16.2 \%$ respectively; $\mathrm{p}=0.038)$ were found to be significantly higher in the patient group compared to the control group as shown in table 1 .
Hypothyroidism group had higher levels of irisin compared to the control group $(2.77 \mathrm{ng} / \mathrm{mL} v s$. $2.15 \mathrm{ng} / \mathrm{mL}$ respectively; $\mathrm{p}=0.017)$. Hypothyroidism group had higher median levels of irisin in obese patients than those in the control group $(3.10 \mathrm{ng} / \mathrm{mL}$ vs. $2.10 \mathrm{ng} / \mathrm{mL}$ relatively; $\mathrm{p}=0.013$ ) (Figure 1 ). In addition, male patients had higher median levels of irisin compared to females $(5.84 \mathrm{ng} / \mathrm{mL}$ vs. $2.46 \mathrm{ng} / \mathrm{mL}$; $\mathrm{p}=0.008$ ).

Table 1. Demographical characteristics and laboratory findings of the study groups

\begin{tabular}{lccc}
\hline Variables & Hypothyroidism & Control & P \\
\cline { 2 - 3 } Sex (male), $\mathrm{n}(\%)$ & $\mathbf{( n = 3 7 )}$ & $\mathbf{( n = 3 7 )}$ & 0.358 \\
Age (years) & $4(10.8)$ & $1(2.7)$ & 0.310 \\
Systolic BP (mmHg) & $32.27 \pm 8.7$ & $34.76 \pm 11.9$ & $0.004^{*}$ \\
Diastolic BP (mmHg) & $127.38 \pm 9.7$ & $120.22 \pm 11.2$ & $0.006^{*}$ \\
BMI (kg/m²) & $65.97 \pm 8.5$ & $60.0 \pm 9.7$ & 0.148 \\
Obesity, n(\%) & $27.77 \pm 5.4$ & $26.11 \pm 4.3$ & $0.038^{*}$ \\
Irisin (ng/mL) & $15(40.5)$ & $6(16.2)$ & $0.013^{*}$ \\
Irisin (ng/mL) & $3.10(1.95-20.2)$ & $2.10(1.64-2.67)$ & $0.017^{*}$ \\
TSH ( $\mu$ llU/mL) & $2.77(1.74-20.19)$ & $2.15(1.64-9.33)$ & $<0.001^{*}$ \\
fT 4 (ng/dL) & $13.1(4.68-100)$ & $1.74(0.5-3.6)$ & $<0.001^{*}$ \\
Anti-TG (IU/mL) & $0.68 \pm 0.2$ & $1.14 \pm 0.1$ & $<0.001^{*}$ \\
Anti-TPO (IU/mL) & $335.8(25-4000)$ & $10.8(4-95.2)$ & $<0.001^{*}$ \\
Total cholesterol (mg/dL) & $7.0(2-17.24)$ & 0.356 \\
Triglyceride (mg/dL) & $238.8(9.12-1000)$ & $176.54 \pm 22.1$ & 0.072 \\
LDL (mg/dL) & $185.08 \pm 35.8$ & $91(41-268)$ & 0.500 \\
HDL (mg/dL) & $114(49-346)$ & $100.65 \pm 23.2$ & 0.550 \\
\hline
\end{tabular}

Continuous variables are expressed as mean $\pm \mathrm{SD}$ or median (min-max). Categorical variables are expressed as $\mathrm{N}(\%)$. * $p<0.05$.

BP: blood pressure; BMl: body mass index; TSH: thyroid stimulating hormone; fT4: free thyroxin; anti-TG: anti-thyroglobulin; anti-TPO: anti-thyroid peroxidase; LDL: low density lipoprotein; HDL: high density lipoprotein.
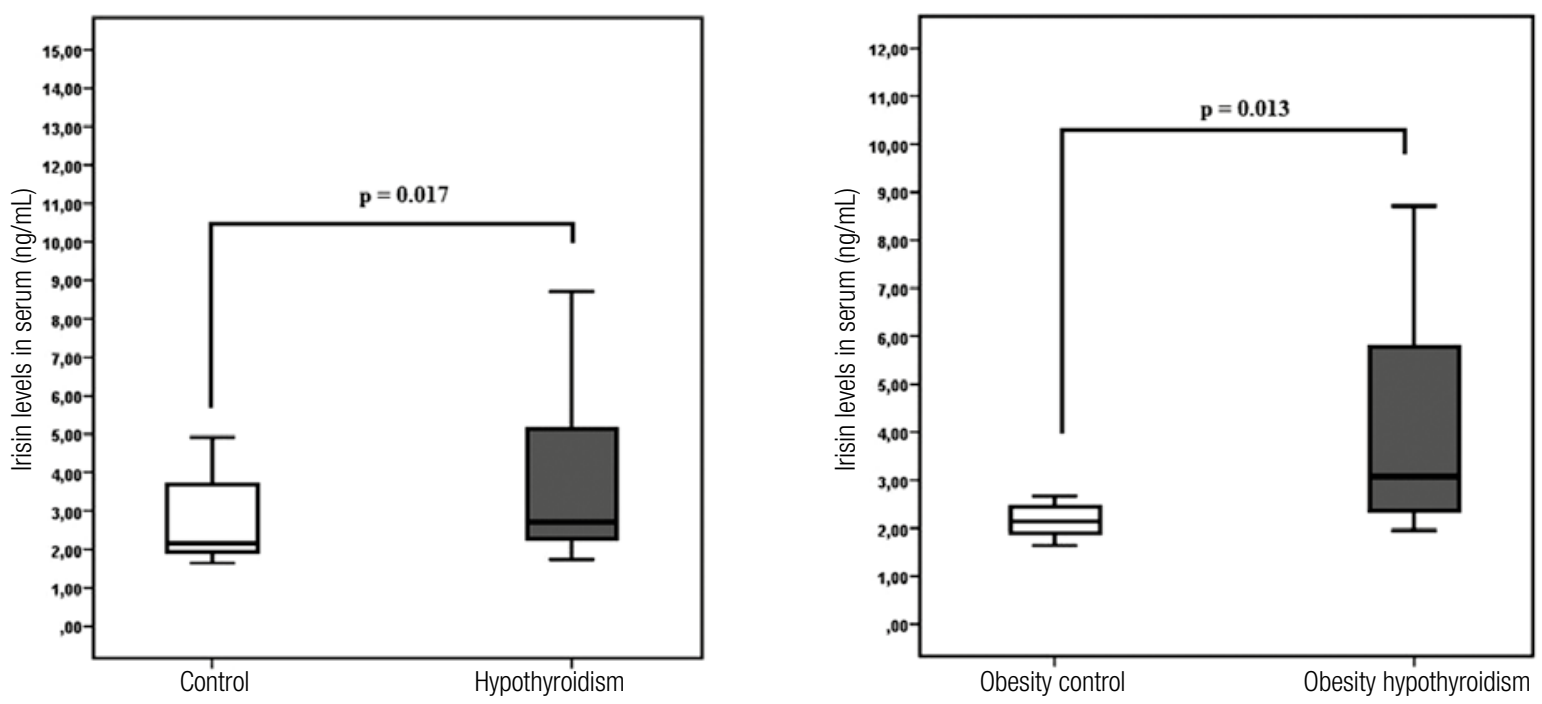

Figure 1. Serum irisin concentration in the all population and obesity population. 
Irisin level was negatively correlated with the age in the whole population and in hypothyroidism group $(\mathrm{r}=-0.255, \mathrm{p}=0.028 ; \mathrm{r}=-0.346, \mathrm{p}=0.036$ respectively). Irisin level was positively correlated with TSH $(\mathrm{r}=0.247, \mathrm{p}=0.034)$ but negatively correlated with fT4 $(\mathrm{r}=-0.316, \mathrm{p}=0.006)$ in the whole population. No correlation was found between other laboratory findings and irisin level in the whole population or in hypothyroidism group (Table 2 ).

Increased systolic and diastolic blood pressures, obesity, high level of TSH, low level of fT4, anti-TG,
anti-TPO, and increased levels of irisin alone were independent risk factors for hypothyroidism. Obesity, fT4 and irisin level were identified to be independent predictors of hypothyroidism in the model including risk factors that were found to be significant in the univariable logistic regression analysis.

We observed that obesity increased the risk for hypothyroidism by 4.420 times and increased irisin levels increased the risk for hypothyroidism by 1.114 times when the other risk factors were kept constant (Table 3).

Table 2. Parameters associated with irisin levels

\begin{tabular}{|c|c|c|c|c|c|c|}
\hline \multirow{2}{*}{ Variables } & \multicolumn{2}{|c|}{ Whole population } & \multicolumn{2}{|c|}{ Control } & \multicolumn{2}{|c|}{ Hypothyroidism } \\
\hline & $\mathbf{r}$ & $\mathbf{P}$ & $\mathbf{r}$ & $\mathbf{P}$ & $\mathbf{r}$ & $\mathbf{p}$ \\
\hline Age (years) & $-0,255$ & $0,028^{*}$ & $-0,143$ & 0,399 & $-0,346$ & $0,036^{*}$ \\
\hline Systolic BP (mmHg) & 0,041 & 0,732 & $-0,015$ & 0,928 & $-0,072$ & 0,672 \\
\hline Diastolic BP (mmHg) & 0.139 & 0.237 & 0.183 & 0.278 & -0.068 & 0.688 \\
\hline $\mathrm{BMI}\left(\mathrm{kg} / \mathrm{m}^{2}\right)$ & 0.028 & 0.811 & -0.009 & 0.959 & 0.058 & 0.732 \\
\hline TSH $(\mu l U / m L)$ & 0.247 & $0.034^{*}$ & -0.143 & 0.397 & 0.161 & 0.341 \\
\hline fT 4 (ng/dL) & -0.316 & $0.006^{*}$ & -0.177 & 0.295 & -0.144 & 0.395 \\
\hline Anti-TG (IU/mL) & 0.193 & 0.100 & -0.052 & 0.760 & -0.093 & 0.583 \\
\hline Anti-TPO (IU/mL) & 0.220 & 0.060 & 0.138 & 0.416 & -0.188 & 0.264 \\
\hline Total cholesterol (mg/dL) & 0.164 & 0.163 & 0.057 & 0.736 & 0.253 & 0.131 \\
\hline Triglyceride (mg/dL) & 0.090 & 0.447 & 0.004 & 0.980 & 0.141 & 0.406 \\
\hline LDL (mg/dL) & 0.207 & 0.077 & 0.090 & 0.595 & 0.297 & 0.074 \\
\hline $\mathrm{HDL}(\mathrm{mg} / \mathrm{dL})$ & 0.013 & 0.913 & 0.040 & 0.816 & 0.104 & 0.539 \\
\hline
\end{tabular}

r: Spearman's rank correlation coefficient.

${ }^{*} p<0.05$

BP: blood pressure; BMl: body mass index; TSH: thyroid stimulating hormone; fT4: free thyroxin; anti-TG: anti-thyroglobulin; anti-TPO: anti-thyroid peroxidase; LDL: low density lipoprotein; HDL: high density lipoprotein.

Table 3. Identification of parameters predicting hypothyroidism risk with logistic regression analysis

\begin{tabular}{|c|c|c|c|c|}
\hline \multirow{2}{*}{ Variables } & \multicolumn{2}{|c|}{ Uni-variable } & \multicolumn{2}{|c|}{ Multivariables } \\
\hline & OR (95\% Cl) & $\mathbf{p}$ & OR (95\% Cl) & p \\
\hline Systolic BP (mmHg) & $1,071(1,018-1,126)$ & 0.008 & - & - \\
\hline Diastolic BP (mmHg) & $1,075(1,018-1,135)$ & 0.010 & - & - \\
\hline Obesity & $3,523(1,181-10,510)$ & 0.024 & $4,420(1,148-27,876)$ & 0.034 \\
\hline TSH & $1,252(1,038-1,693)$ & 0.010 & - & - \\
\hline FT 4 & $0,645(0,469-0,887)$ & 0.007 & $0.619(0.425-0.903)$ & 0.013 \\
\hline Anti-TG & $1,075(1,028-1,124)$ & 0.001 & - & - \\
\hline Anti-TPO & $1,683(1,147-2,469)$ & 0.008 & - & - \\
\hline İrisin (ng/mL) & $1,138(1,058-1,351)$ & 0.021 & $1,114(-1,034-1,592)$ & 0.027 \\
\hline
\end{tabular}

Nagelkerke $\mathrm{R} 2=0.55, \mathrm{p}<0.05$.

BP: blood pressure; TSH: thyroid stimulating hormone; fT4: free thyroxin; anti-TG: anti thyroglobulin; anti-TPO: anti-thyroid peroxidase. 


\section{DISCUSSION}

We found that serum irisin levels were higher in the hypothyroidism group compared to control group in our study. Similarly, serum irisin levels of obese patients in the hypothyroidism group was detected to be higher compared to the obese patients in the healthy control group. In addition, we observed that serum irisin level was increased in correlation with increased severity of hypothyroidism (with increased TSH and decreased fT4). To the best of our knowledge, this study is the first to investigate the relationship between the serum irisin level and TFT in HT patients with hypothyroidism.

Many recent studies have focused on the relationship of obesity to the hormones synthesized in the central nervous system or by peripheral endocrine organs (7). Some hormones cause an increase in the mass of fatty tissue by stimulating adipogenesis with a direct effect on adipose tissue while the others act on the central nervous system, causing an energy intake higher than consumed, and hence obesity (8).

Irisin is a hormone that was discovered a few years ago and known to replace the white adipose tissue with brown adipose tissue, showing an anti-obesity effect $(12,13)$. It shows its effect in autocrine and paracrine manner similarly to other locally synthesized hormones (14). Another current review has underlined that irisin and similar hormones with local effects function without losing their contact with central endocrine organs (e.g., pancreas, thyroid) (8).

Irisin level was identified to be high in insulin resistance (15), metabolic syndrome (16), and obesity and to be low in anorexia nervosa (6), chronic renal failure (17), and type 2 diabetes mellitus (18).

Two different studies conducted with different objectives on a study group without any thyroid disease investigated the relationship between irisin levels and TFT. Stengel and cols. investigated the relationship between irisin levels and TFT in their study in patients with obesity and anorexia nervosa, and the relationship between TFT and irisin was not statistically significant in either of the groups (6). Gouni-Berthold and cols. also examined the relationship between irisin levels and TFT on 72 male patients, who used lipid-lowering medicine, and found no statistically significant relationship either in the groups or in the whole population (19). The results of their study are in consistence with our results since their study was performed with patients without euthyroid or another documented thyroid disease.
In our study, patients with hypothyroidism and obese hypothyroidism had higher levels of irisin compared to the healthy control group. There was a positive correlation between serum irisin levels and TSH while a negative correlation was found between irisin levels and fT4.

Results of our study can be deemed to be very consistent considering the apparent pathogenic mechanisms. TSH level, which is increased in hypothyroidism, causes the differentiation of preadiposites through the receptors in the preadipose tissue and induces adipogenesis. Previously formed adiposites become hypertrophic with adipogenesis and the newly formed adiposities undergo hyperplasy, resulting in increased adipose tissue. Hormones such as leptine, ghrelin, NUCB2/nesfatin-land irisin can be synthesized in order to keep the fat distribution in balance in the increased white adipose tissue (20). In such case, irisin levels can be found to be high with increasing TSH levels.

Another perspective can be the fact that FNDC5, which is found in the thyroid tissue and a precursor of irisin (10), is destructed by chronic inflammation of thyroid follicular in HT and goes into the blood stream, resulting in increased levels of irisin.

We also observed in our study that irisin levels of males were higher compared to females, and irisin levels decreased with aging. This suggests that it might be associated with the total skeletal mass.

To the best of our knowledge, this study is the first in literature to identify that obesity, irisin level and fT4 level are independent risk factors for hypothyroidism.

The limitations of our study include the inadequate number of patients, low percentage of male participants, and unknown irisin levels of hypothyroidism patients with high levels of TSH after being treated with levothyroxine.

\section{CONCLUSIONS}

As a result, further studies investigating the relationship of irisin hormone, which has newly been discovered and relationship of which with other hormones remains unknown, with the central and peripheral endocrine organs are needed. We believe that if the anti-obesity effect of irisin can be fully elucidated, it would be a very important step for understanding the unknown mechanisms of obesity, the disease of the current age.

Acknowledgement: we would like to thank Dr. Nihal Özkayar for critical reading of this manuscript. 
Disclosure: no potential conflict of interest relevant to this article was reported.

\section{REFERENCES}

1. Li D, Cai W, Gu R, Zhang Y, Zhang H, Tang K, et al. Th17 cell plays a role in the pathogenesis of Hashimoto's thyroiditis in patients. Clin Immunol. 2013;149(3):411-20.

2. Sorisky A, Bell A, Gagnon A.TSH receptor in adipose cells. Horm Metab Res. 2000;32(11-12):468-74.

3. Nishimura S, Manabe I, Nagasaki M, Hosoya $Y$, Yamashita H, Fujita $\mathrm{H}$, et al. Adipogenesis in obesity requires close interplay between differentiating adipocytes, stromal cells, and blood vessels. Diabetes. 2007;56(6):1517-26.

4. Feldt-Rasmussen U. Thyroid and leptin. Thyroid. 2007;17(5):413-9.

5. Boström P, Wu J, Jedrychowski MP, Korde A, Ye L, Lo JC, et al. A PGC1- $\alpha$-dependent myokine that drives brown-fat-like development of white fat and thermogenesis. Nature. 2012;481(7382):463-8.

6. Stengel A, HofmannT, Goebel-Stengel M, Elbelt U, Kobelt P, Klapp BF. Circulating levels of irisin in patients with anorexia nervosa and different stages of obesity--correlation with body mass index. Peptides. 2013;39:125-30.

7. Contreras C, Gonzalez F, Ferno J, Dieguez C, Rahmouni K, Nogueiras $R$, et al. The brain and brown fat. Ann Med. 2014:1-19.

8. Aydin S.Three new players in energy regulation: preptin, adropin and irisin. Peptides. 2014;56:94-110.

9. Castillo-Quan Jl. From white to brown fat through the PGC-1 $\alpha$ dependent myokine irisin: implications for diabetes and obesity. Dis Model Mech. 2012;5(3):293-5.

10. Huh JY, Panagiotou G, Mougios V, Brinkoetter M, Vamvini MT, Schneider BE, et al. FNDC5 and irisin in humans: I. Predictors of circulating concentrations in serum and plasma and II. mRNA expression and circulating concentrations in response to weight loss and exercise. Metabolism. 2012;61(12):1725-38.
11. Friedewald WT, Levy RI, Fredrickson DS. Estimation of the concentration of low-density lipoprotein cholesterol in plasma, without use of the preparative ultracentrifuge. Clin Chem. 1972;18(6):499-502.

12. Roca-Rivada A, Castelao C, Senin LL, Landrove MO, Baltar J, Belén Crujeiras $A$, et al. FNDC5/irisin is not only a myokine but also an adipokine. PLoS One. 2013;8(4):e60563.

13. Zhang Y, Li R, Meng Y, Li S, Donelan W, Zhao Y, et al. Irisin stimulates browning of white adipocytes through mitogen-activated protein kinase p38 MAP kinase and ERK MAP kinase signaling. Diabetes. 2014;63(2):514-25.

14. Aydin S, KulogluT, Aydin S, Eren MN, Celik A, Yilmaz M, et al. Cardiac, skeletal muscle and serum irisin responses to with or without water exercise in young and old male rats: cardiac muscle produces more irisin than skeletal muscle. Peptides. 2014;52:6873.

15. Sesti G, Andreozzi F, Fiorentino TV, Mannino GC, Sciacqua A, Marini MA, et al. High circulating irisin levels are associated with insulin resistance and vascular atherosclerosis in a cohort of nondiabetic adult subjects. Acta Diabetol. 2014;51(5):705-13.

16. Park KH, Zaichenko L, Peter P, Davis CR, Crowell JA, Mantzoros CS. Diet quality is associated with circulating $\mathrm{C}$-reactive protein but not irisin levels in humans. Metabolism. 2014;63(2):233-41.

17. Ebert T, Focke D, Petroff D, Wurst U, Richter J, Bachmann A, et al. Serum levels of the myokine irisin in relation to metabolic and renal function. Eur J Endocrinol. 2014;170(4):501-6.

18. ChoiYK, Kim MK, Bae KH, Seo HA, Jeong JY, LeeWK, et al. Serum irisin levels in new-onset type 2 diabetes. Diabetes Res Clin Pract. 2013;100(1):96-101.

19. Gouni-Berthold I, Berthold HK, Huh JY, Berman R, Spenrath N, Krone W, et al. Effects of lipid-lowering drugs on irisin in human subjects in vivo and in human skeletal muscle cells ex vivo. PLoS One. 2013;8(9):e72858.

20. Dua A, Hennes MI, Hoffmann RG, Maas DL, Krakower GR, Sonnenberg GE, et al. Leptin: a significant indicator of total body fat but not of visceral fat and insulin insensitivity in African-American women. Diabetes. 1996;45(11):1635-7. 